\title{
Reduced Variance using ADVANTG in Monte Carlo Calculations of Dose Coefficients to Stylized Phantoms
}

\author{
Mauritius Hiller ${ }^{1, a}$, Michael Bellamy ${ }^{1}$, Keith Eckerman ${ }^{1}$, Nolan Hertel ${ }^{1,2}$ \\ ${ }^{1}$ Oak Ridge National Laboratory, Center for Radiation Protection Knowledge, P.O. Box 2008 MS 6335, Oak Ridge, TN 37831 - \\ 6335 \\ ${ }^{2}$ Georgia Institute of Technology, 770 State Street, Atlanta, GA 30332-0745
}

\begin{abstract}
The estimation of dose coefficients of external radiation sources to the organs in phantoms becomes increasingly difficult for lower photon source energies. This study focus on the estimation of photon emitters around the phantom. The computer time needed to calculate a result within a certain precision can be lowered by several orders of magnitude using ADVANTG compared to a standard run. Using ADVANTG which employs the DENOVO adjoint calculation package enables the user to create a fully populated set of weight windows and source biasing instructions for an MCNP calculation.
\end{abstract}

\section{Introduction}

The calculation of dose coefficients to human phantoms for Monte Carlo calculations sets a huge demand on available computing power. Efforts that help reduce the variance of the calculation and increase the efficiency greatly facilitate the solution of such problems.

While the applicability of standard variance reduction methods implemented in MCNP is limited and, especially at low energies, the time and effort required to create appropriate weight windows is excessive. However, the ADVANTG (1) code can create weight windows in a short time frame.

In this paper, an example the use of the ADVANTAG code to increase both the speed and efficiency of an MCNP6 computation of organ doses for a stylized phantom fully emerged in contaminated water is presented.

\section{Methods}

The calculations shown herein focus on the determination of the dose coefficient for the adrenals, a small organ, inside the body that presents a particular challenge to generate statistically acceptable uncertainties when it is used as a tally cell. Computations were performed and presented for photons in an energy range from $10 \mathrm{keV}$ to $500 \mathrm{keV}$. The calculations were terminated at an error of $3 \%$ in the adrenals.

The phantom used for the calculations is the ORNL stylized adult phantom.

The calculations were performed on an Intel core i7 CPU with 4 cores. MCNP 6.1.1 (2) was used with the OMP multi core support using the "task" setting.

\subsection{Situation Prior to Optimization}

The stylized phantom is fully immersed in water. The water is in a cubical box with $4 \mathrm{~m}$ side length, whereas the adult male is about $80 \mathrm{~cm}$ wide and $180 \mathrm{high}$ and has been centred in the box. All water is considered contaminated with photon emitters so the phantom is irradiated from all sides. The simulated photon energy range is from $10-500 \mathrm{keV}$.

\subsection{ADVANTG Setup}

The ADVANTG code (Mosher et al. 2015) which creates a weight window input file for MCNP using the Denovo code was used in this problem. Denovo uses a multigroup adjoint calculation to determine the weight windows. This makes it possible to create completely assigned weight windows even for a fine mesh at low energies in a short time. Figure 1 shows a set of weight windows generated by ADVANTG, were regions of highest interest are shown in blue while regions of least interest are shown in red.

Besides the weight windows, ADVANTG also provides a source bias setting.

An MCNP input file was produced with ADVANTAG determined weight windows. Then the run of the optimized input file was stopped when an error of $3 \%$ in the adrenals was achieved.

\section{Results}

The CPU time needed to run ADVANTG to create the modified MCNP input file and the CPU time then needed to run the MCNP file were summed and are compared to the results from MCNP runs which did not use any variance reduction techniques in Table 1.

\footnotetext{
${ }^{\mathrm{a}}$ Corresponding author: hillermm@ornl.gov
} 

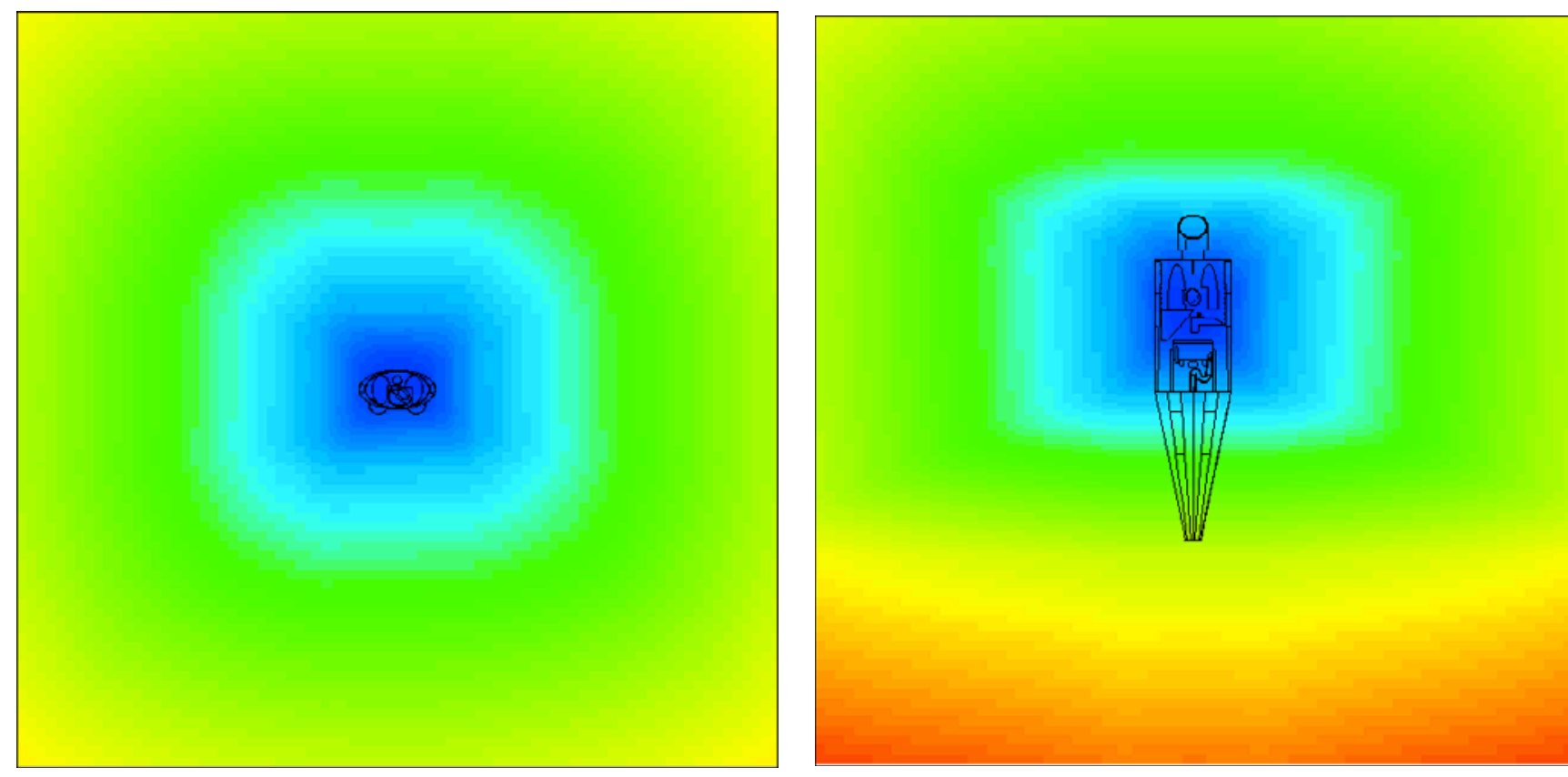

Figure 1: Front and top view of the phantom with the weight windows assigned by ADVANTG. Areas of high importance are shown in blue areas of low importance in red.

Figure 2 shows the run time of the unmodified run against the run time of the ADVANTG code plus the modified run. The time gained using ADVANTG can be seen as the distance from the top of the bar of the ADVANTG run to the line of the unmodified runs.

It can be see seen that ADVANTG helps to significantly speed up the runs by for lower energies. The time needed to run the Denovo code is relatively constant with the energy. Therefore, the effect on the speed of the computation of ADVANTG is less towards higher energies, in fact the direct runs are faster for energies above $100 \mathrm{keV}$.

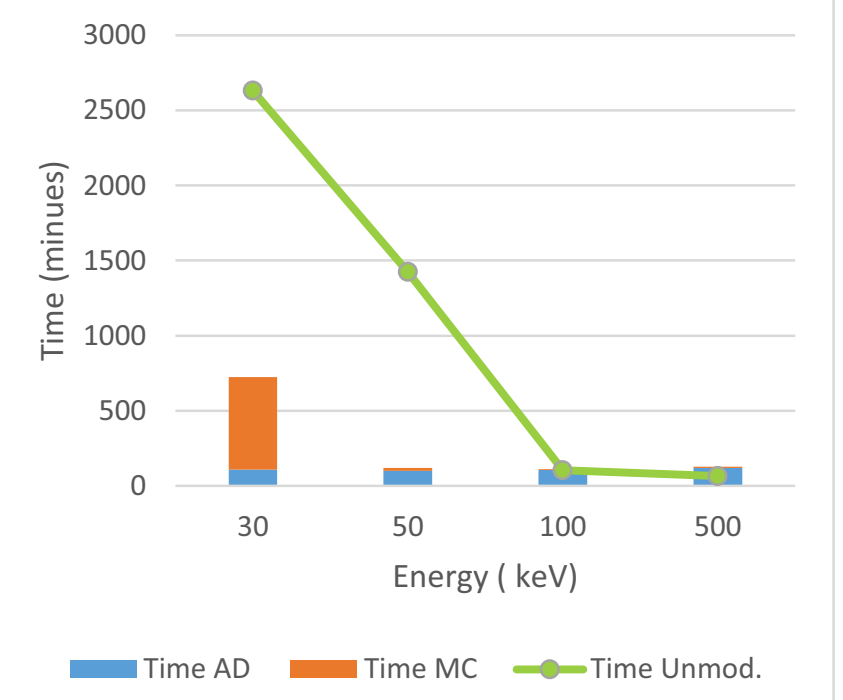

Figure 2: Run-times of unmodified MCNP runs against ADVANTG plus modified MCNP runs.

\section{Discussion}

The reduced efficiency towards higher energy calculations can be explained with the time needed to create the optimizations in ADVANTG. For higher energies, the runs without optimization finish in a time, shorter than the ADVANTG run, thus it becomes unfavourable to optimize the problem with ADVANTG prior to its MCNP run. Photons with energies above $100 \mathrm{keV}$ more easily penetrate the phantom and reach the inner organs.

\section{References}

1. W. Mosher, S. R. Johnson, A. M. Bevill, A. M. Ibrahim, C. R. Daily, T. M. Evans, J. C. Wagner, J. O. Johnson, R. E. Grove. ADVANTG - An Automated Variance Reduction Parameter Generator. ORNL/TM-2013/416 Rev. 1, Oak Ridge National Laboratory, Oak Ridge, TN, 2015.

2. D. B. Pelowitz, A. J. Fallgren, G. E. McMath. MCNP6 User's Manual, Code Version 6.1.1beta. LA-CP-14-00745, Rev. 0, 2014. 
Table 1: Run-times for Monte Carlo runs with and without prior optimization in ADVANTG.

\begin{tabular}{|c|c|c|c|c|c|c|c|}
\hline \multirow[b]{2}{*}{$\mathrm{E}(\mathrm{keV})$} & \multicolumn{2}{|c|}{ Unmodified } & \multicolumn{3}{|c|}{ ADVANTG } & \multicolumn{2}{|c|}{ Unmodified / ADVANTG } \\
\hline & $\begin{array}{r}\text { Time MC } \\
(\min )\end{array}$ & $\begin{array}{r}\text { Mean } \\
(\mathrm{MeV} / \mathrm{g})\end{array}$ & $\begin{array}{r}\text { Time AD } \\
\text { (min) }\end{array}$ & $\begin{array}{r}\text { Time MC } \\
(\min )\end{array}$ & $\begin{array}{r}\text { Mean } \\
(\mathrm{MeV} / \mathrm{g})\end{array}$ & Time & Mean \\
\hline 500 & 65.9 & $3.947 \mathrm{E}-09$ & 120 & 8 & $3.92 \mathrm{E}-09$ & 0.51 & 1.01 \\
\hline 100 & 105.0 & $5.543 E-10$ & 105 & 4 & 5.67E-10 & 0.96 & 0.98 \\
\hline 50 & 1425.4 & $1.44 \mathrm{E}-10$ & 101 & 18 & $1.48 \mathrm{E}-10$ & 12.02 & 0.97 \\
\hline 30 & 2633.3 & $2.15 \mathrm{E}-11$ & 108 & 617 & $2.15 E-11$ & 3.63 & 1.00 \\
\hline
\end{tabular}

No convergence in reasonable time frame of several days of calculation 